\title{
Cardiovascular Responses to Adequate Stimuli in Essential Hypertension
}

\author{
By
Ryuji Shioji, Hidenori Takahashi, Hikoyuki Suzuki
and Masataka Kato \\ From the Medical Clinic of Prof. T. Torikai, Tohoku \\ University School of Medicine, Sendai
}

(Received for publication, July 7, 1962)

In 1934, Keith et al. ${ }^{1)}$ divided cases of essential hypertension into four groups and clinically studied their course and prognosis. They concluded that there was a marked variation in the clinical course, length of life and pathologic lesions of hypertensive patients. Since they reported on their pioneer works, this classification of essential hypertension had been used all over the world.

According to the international classification, ${ }^{2)}$ essential hypertension has been classified into 3 types, as follows:

1) essential benign hypertension

2) hypertension with arteriolar nephrosclerosis

3) essential malignant hypertension

More or less different systems of classification have been published from different standpoints, but it seems that of Keith-Wagener is the best for general use.

Though the pathogenesis of essential hypertension is not yet clear, hypertension can be considered as a disease of cardiovascular system and as due to the adaptation of the heart to the task of maintaining normal cardiac output against increased vascular resistance.

All past systems of the classification were made from a point of view of morphological findings in the peripheral arterioles, but not on the basis of functional observation of the cardiovascular system in essential hypertension.

In the present experiment, the cardiovascular responses to adequate stimuli were studied in essential hypertension and some interesting results were obtained, as follows.

\section{METHODS}

Thirty-four normal subjects free of any sign of cardiovascular abnormality, aged from 24 to 67 years and twenty-four hypertensive patients, whose ages ranged

塩路隆治, 高橋秀典, 鈴木彦之, 加藤政孝 
from 38 to 62 years, were studied in this experiment. Respiration and changes in posture (from recumbent to standing position) were applied as stimuli.

The subjects were made to alter their position actively from recumbent to standing position after they had resumed the recumbent posture for 30 minutes. Throughout the procedure the subjects were securely supported to maintain their positions and bipolar chest lead was used in order to exclude the effect of any muscular activity.

In our experiment reported here, every change of instantaneous cardiac rhythm was recorded continuously by means of a special type of instrument, Matsuda's Cardiotachograph, ${ }^{3)}$ over a relatively long period of time.

Cardiotachograms were taken continuously for 5-7 minutes in each position (in recumbent and standing positions, respectively), always the respiration being recorded simultaneously. ${ }^{4}$ Samples of such cardiotachograms are represented in Fig. 1.

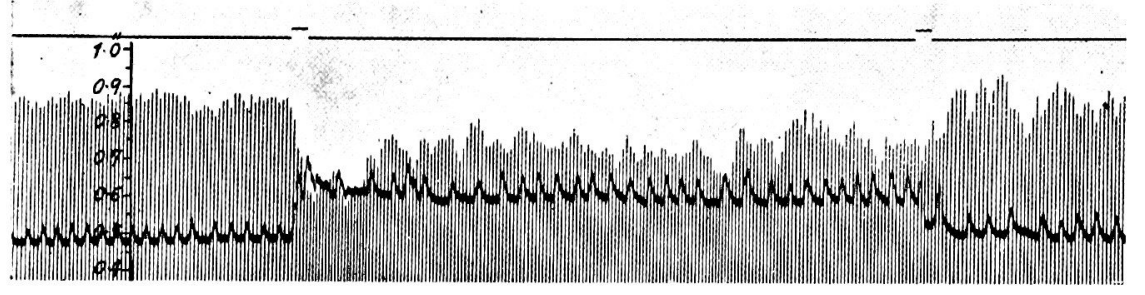

Fig. 1. Cardiotachogram of normal subject. Effect of change in posture.

Upper: signal. Middle: cardiotachogram, pulse interval in second can be read in the scale at left. Lower: respiration, inspiration upward.

Subjects were made to alter their positions actively from horizontal to standing at the first signal and were returned to the original position at the sencond signal.

While the instantaneous cardiac rate was recorded by this cardiotachograph continuously throughout the experiment, the blood pressure was measured twice in each position, by the ordinary cuff-auscultation method on the right brachial artery approximately at the height of the heart, the mean value of the two measurements being taken as the blood pressure in each position.

The extent of respiratory arrhythmia, phasic variation of the instantaneous cardiac rhythm, synchronous with respiratory movement, was expressed quantitatively by the maximum difference between the expiratory and inspiratory cardiac interval. Here the expiratory cardiac interval means the average of two values for the expiratory phases preceding and following the inspiration. Individual respiratory arrhythmia was indicated by the mean value of 20 respiratory arrhythmias.

The variation coefficient of cardiac rate and blood pressure advocated by Suzuki $^{5}$ ) was calculated by the following formula from the cardiotachograms and 
blood pressure, respectively.

Variation coefficient of cardiac rate $=$ $\left|\frac{\text { cardiac rate in recum. pos. - cardiac rate in stand. pos. }}{\text { cardiac rate in recum. pos. }}\right|$

Variation coefficient of blood pressure $=$

systolic blood pressure in recum. pos. - systolic blood pressure in stand. pos. systolic blood pressure in recum. pos.

\section{RESULTS}

1) Blood pressure and respiratory arrhythmia.

The relation between the systolic blood pressure and the extent of respiratory arrhythmia in normal subjects and hypertensive patients is illustrated in Fig. 2. There was a remarkable negative correlation between the systolic blood pressure and the respiratory arrhythmia in normal subjects, that is, the higher the former, the smaller was the latter, but no prominent relation was observed in hypertensive patients. Hypertensive patients showed a small and nearly constant value of respiratory arrhythmia independent of the systolic blood pressure. Matsuda and Suzuki') reported that the respiratory arrhythmia was generally in close connection with cardiac interval and age.

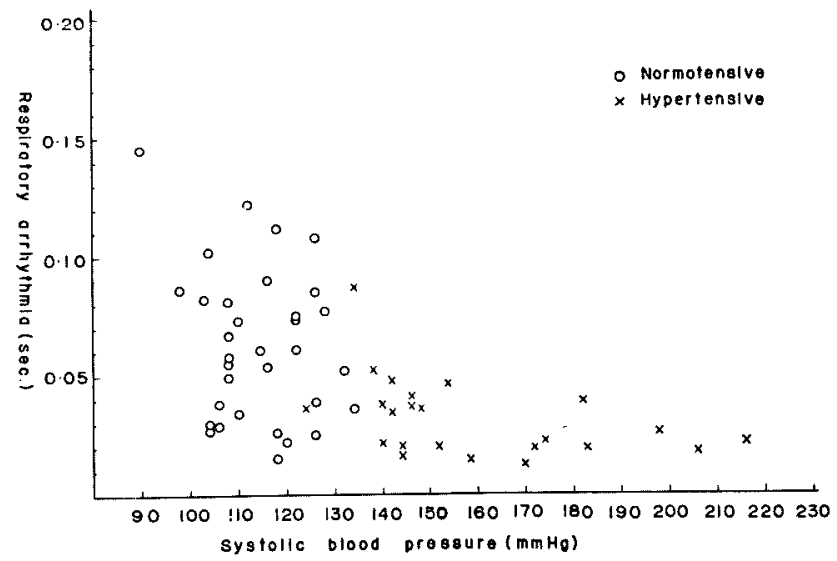

Fig. 2. Relationship between systolic blood pressure and respiratory arrhythmia.

In the previous communication, Kato et al. ${ }^{7}$ confirmed that the respiratory arrhythmia was smaller in hypertensive patients than in normal subjects, even if the influence of age was taken into consideration, when the respiratory arrhythmia of normal subjects as well as of hypertensive patients was plotted against the ages of individuals. 
Our present results are consistent with the results of the above-named investigators (Matsuda et al. and Kato et al.).

The relation between the diastolic blood pressure, the pulse pressure and the respiratory arrhythmia in normal and hypertensive patients is shown in Figs. 3 and 4. In normal subjects, there was no distinct, but a considerable negative correlation between them.

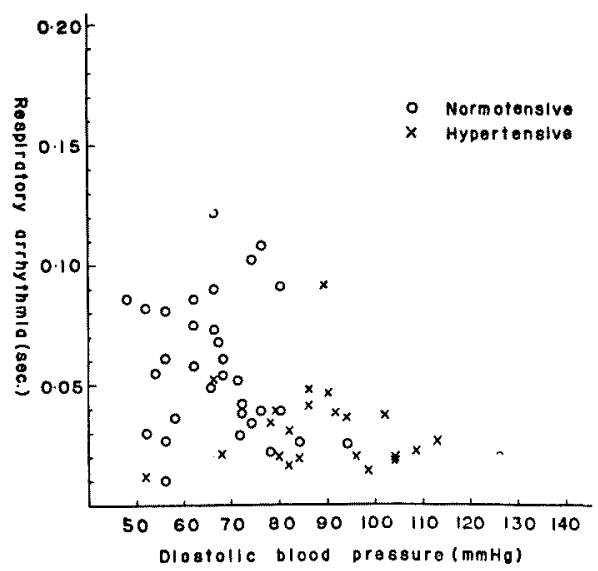

Fig. 3. Relationship between diastolic blood pressure and respiratory arrhythmia.

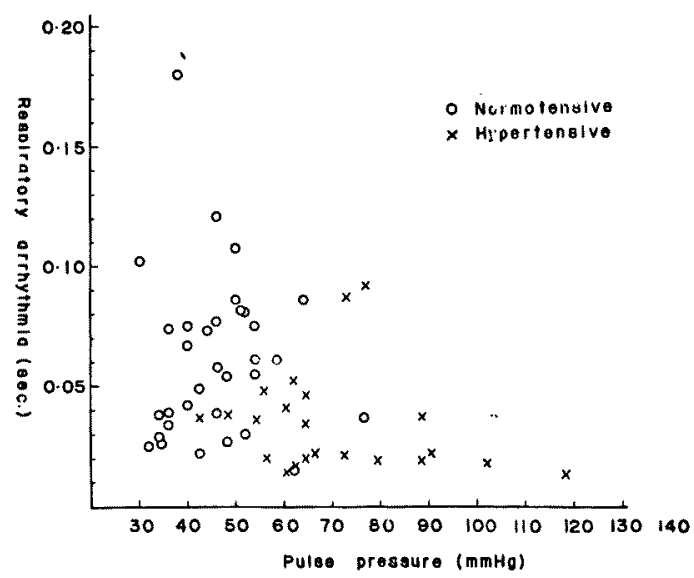

Fig. 4. Relationship between pulse pressure and respiratory arrhythmia.

2) Variation coefficient of cardiac rate and blood pressure.

The subjects were made to alter their position from recumbent to standing 
position actively after they had resumed the recumbent posture for 30 minutes. Cardiotachograms were taken continuously for 5-7 minutes and blood pressure was taken twice in each position. The cardiac rate in each position was calculated from the cardiotachograms. As mentioned above, the variation coefficient of cardiac rate was the absolute value of the difference of cardiac rate in recumbent and standing position divided by the cardiac rate in recumbent position. The variation coefficient of blood pressure was similarly the absolute value of the difference of systolic blood pressure in recumbent and standing position divided by the systolic blood pressure in recumbent position.

We noticed a distinct difference in the frequency distribution of the values of variation coefficient of cardiac rate and systolic blood pressure, as shown in Figs. 5 and 6 . The variation coefficient of cardiac rate was smaller in hypertensive patients than in normal subjects (Fig. 5). On the contrary, the variation coefficient of systolic blood pressure is greater in hypertensive patients than in normal subjects (Fig. 6).

3) A new representation of cardiac rate and systolic blood pressure in essential hypertension.

As shown in Fig. 7, the cardiac rate and the systolic blood pressure were respectively plotted at the left and the right side on the abscissa, the difference of cardiac rate and systolic blood pressure in the recumbent and standing postures were plotted on the ordinate, upward when they increased and downward when they decreased.

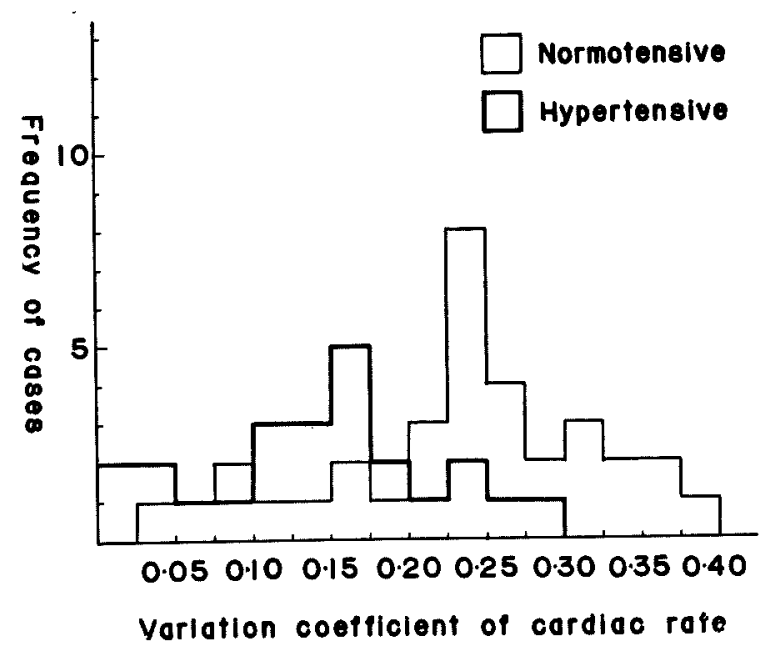

Fig. 5. Frequency distribution of the variation coefficient of cardiac rate in normotensives and hypertensives. 


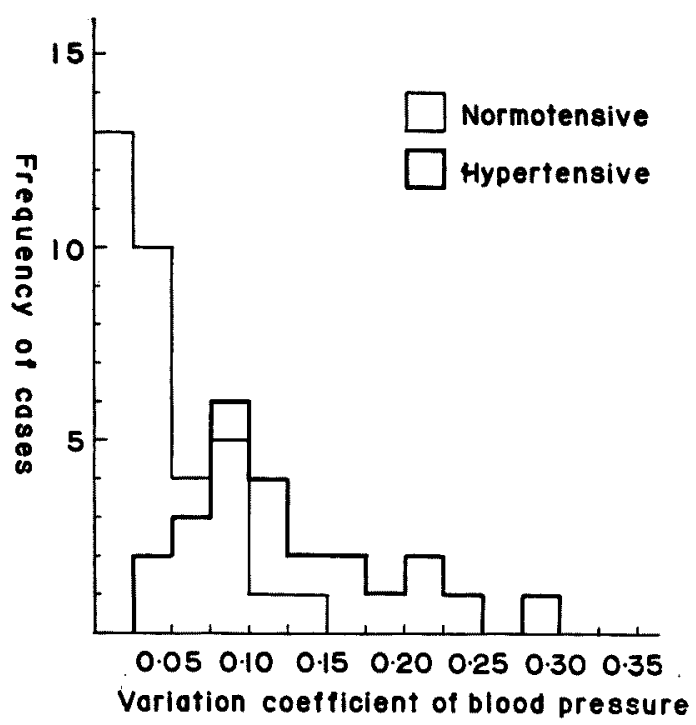

Fig. 6. Frequency distribution of the variation coefficient of systolic blood pressure in normotensives and hypertensives.

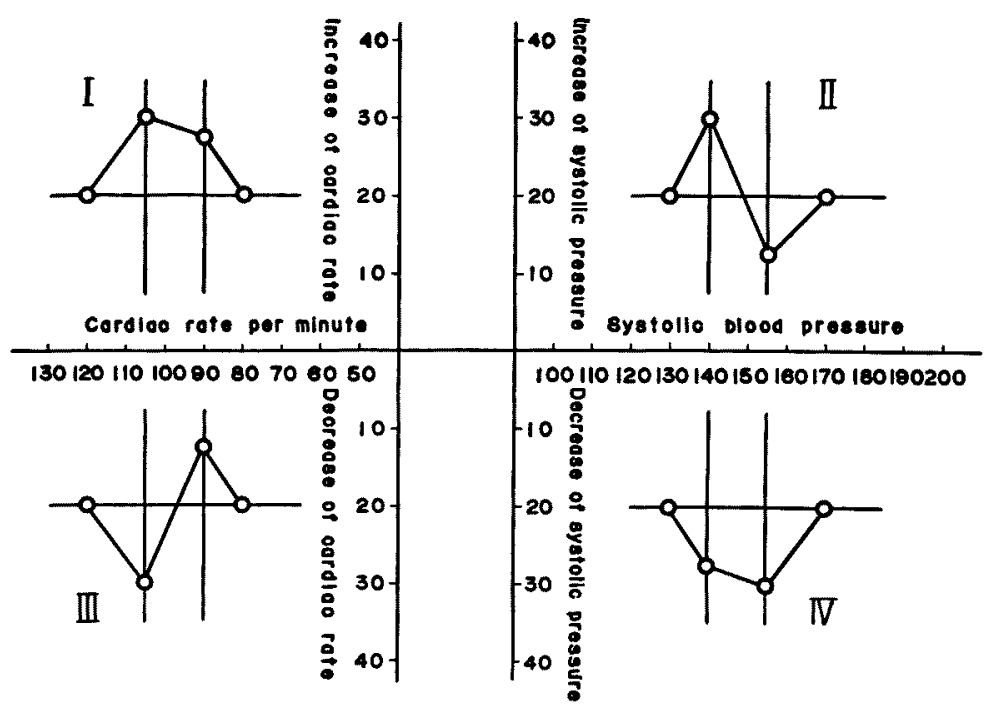

Fig. 7. A new representation of cardiac rate and systolic blood pressure. (see text)

Four combinations were obtained in such a representation. If these combinations are named I, II, III and IV type, the frequency of 34 normal subjects and 24 hypertensive patients belonging to each type is shown in Table I. Almost all the normal subjects as well as hypertensive patients were included in II type, some 
Table I. Distribution of 4 Types

\begin{tabular}{r|c|c|c|c}
\hline Type & I & II & III & IV \\
\hline Normotensive & 5 & 28 & 1 & 0 \\
\hline Hypertensive & 2 & 20 & 0 & 2
\end{tabular}

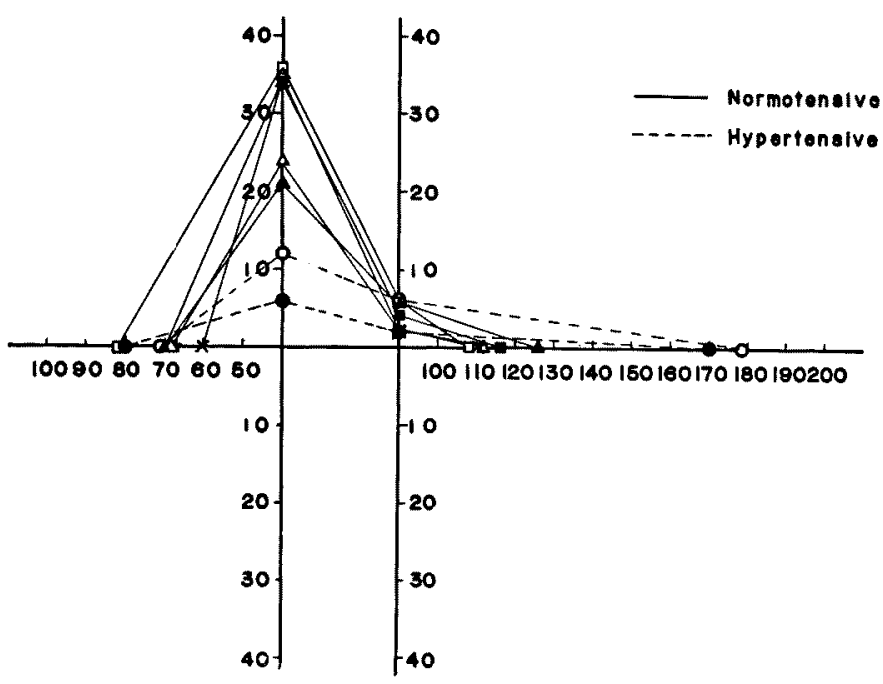

Fig. 8. Curves of normotensives and hypertensives belonging to I type of a new representation of cardiac rate and systolic blood pressure.

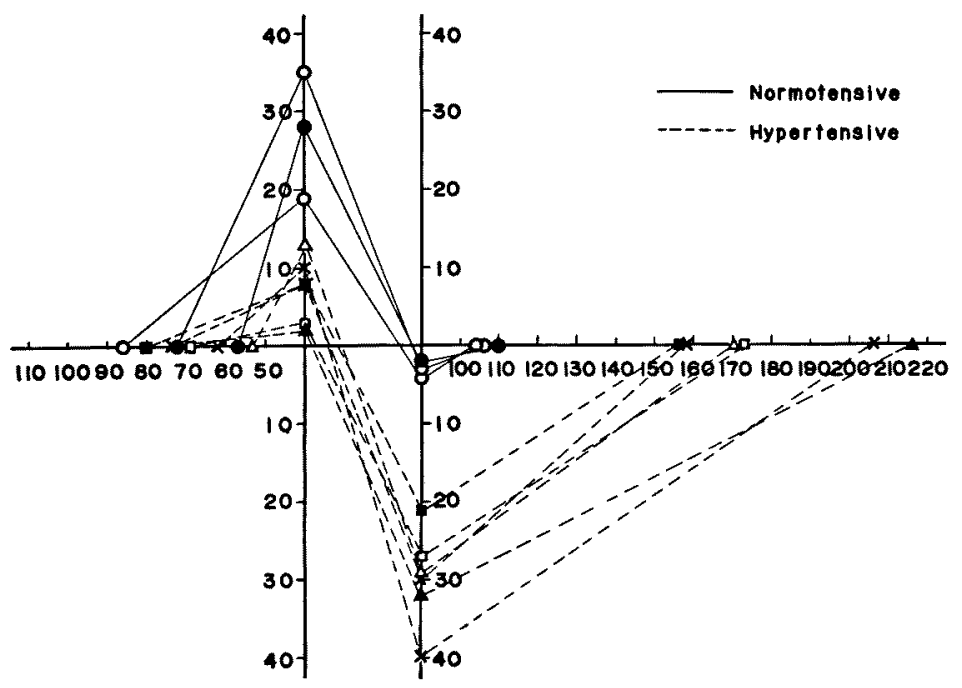

Fig. 9. Curves of normotensives and hypertensives belonging to II type of a new representation of cardiac rate and systolic blood pressure. 
cases in I type, 1 case of normal subjects in III type and 2 cases of hypertensive patients in IV type.

The 2 cases of hypertensive patients who belonged to IV type were of malignant hypertension. Kato et al. ${ }^{7)}$ had already reported that the malignant hypertensive patient showed paradoxical reaction of cardiac rate in postural change. The curves of normal subjects and hypertensive patients belonging to I and II type are illustrated in Figs. 8 and 9.

When we can comprehend that the values of variation coefficient of cardiac rate and systolic blood pressure are in opposite relation in normal subjects and hypertensive patients, it becomes clear that the variation coefficient of cardiac rate is greater in normal than in hypertensive and the variation coefficient of systolic blood pressure is greater in hypertensive than in normal subjects with change of posture.

\section{DISCUSSION}

As reported above, we confirmed in normal subjects and almost all hypertensive patients tested that the resting cardiac rate differed by the posture of the subjects, high in standing, low in recumbent position. Moreover, we concluded that there was a remarkable negative correlation between the systolic blood pressure and the respiratory arrhythmia in normal subjects. The hypertensive patients showed a small and nearly constant value of respiratory arrhythmia independently of the systolic blood pressure. Concerning the difference of resting cardiac rate by posture, we hardly feel it necessary to cite references here, for it is a universally accepted fact.

The higher the systolic blood pressure rose, the smaller was the respiratory arrhythmia in normal subjects. This fact was in accord with Matsuda and Suzuki's result, ${ }^{6)}$ namely, the respiratory arrhythmia was generally in close connection with age, and it was commonly recognized that the older the age, the higher the systolic blood pressure.

The physiological mechanisms responsible for the changes of cardiac rate and blood pressure with change of posture were explained in normal subjects as follow. ${ }^{8)}$ As the central venous pressure must be lowered in the change of posture from the recumbent to the standing position owing to diminished return of venous blood from the dependent part of the body by gravity, the lessened filling of the auricle and/or cava stimulates the sensory ending which calls the reflex, as discovered by Aviado et al., ${ }^{9)}$ into play and causes the acceleration of the heart beat. The same reflex would probably bring about peripheral vaso-constriction through the vasomotor center. Both the tachycardia and the vaso-constriction would contribute to the adjustment for the initial drop of blood pressure and pronounced fluctuation of cardiac rate immediately following the change to standing posture, and are to be interpreted as the effect of both the reflexes coming from the arterial 
side (aortic and carotid sinus) and venous side (vago-pressor-reflex, McDowall ${ }^{10}$ ) and Aviado $\left.{ }^{9}\right)$. However, for the subsequent persistence of the accelerated cardiac rate in the standing posture, the vago-pressor-reflex (McDowall and Aviado) could be the principal mechanism concerned as both pulse pressure and cardiac output remain lowered as long as the standing posture is maintained in spite of the apparently completed compensation of systolic pressure.

In hypertensive patients, the respiratory arrhythmia and the variation coefficient of cardiac rate were smaller and the variation coefficient of blood pressure was larger than that of normal subjects, that is, the cardiac rate varied only a little, even if it increased (Figs. $8 \& 9$ ), on the other hand, the blood pressure lowered eminently in the greater part of cases (Fig. 9) and rose in a few cases (Fig. 8) with change of posture.

Though the physiological mechanism of such findings is not yet clear, it is certain that the reflex route mentioned above may be damaged elsewhere by some unknown mechanism in hypertensive patients.

It is interesting that a paradoxical reaction of cardiac rate was observed in malignant hypertensive patients and they belonged to IV type of the new representation. It seems to show that the type of hypertension shifts from II or I to IV in proportion to the aggravation of the hypertension.

\section{SUMLARY}

The cardiovascular responses to adequate stimuli were studied in 34 normal subjects and 24 hypertensive patients, using respiration and change in posture as stimuli.

Hypertensive patients showed a small and nearly constant value of respiratory arrhythmia independent of the systolic blood pressure and no correlation was found between them, but in normal subjects the higher the systolic blood pressure, the smaller the respiratory arrhythmia.

The variation coefficient of cardiac rate was smaller in hypertensive patients than in normal subjects, but the variation coefficient of blood pressure was greater in hypertensive patients than in normal subjects.

According to our new representation both normal subjects and hypertensive patients were almost all included in I or II type, but only 2 cases of malignant hypertensive patients in IV type.

The authors wish to express their thanks to Prof. T. Torikai for his advice and encouragement.

\section{References}

1) Keith, N.M., Wagener, H.P. \& Barker, N.W., Am. J. Med. Sci., 1939, 197, 332.

2) International Classification of Diseases, World Health Organization, 1955, 1, 149.

3) Matsuda, K., Tohoku J. Exper. Med., 1948, 49, 246. 
4) Kato, M. \& Sato, H., Tohoku J. Exper. Med., 1949, 51, 378.

5) Kushima, K. \& Suzuki, T., Climacteric disturbance and climacteric disturbancelike syndrome (Jap.), Igaku Shoin, 1956.

6) Matsuda, K. \& Suzuki, T., Tohoku J. Exper. Med., 1952, 56, 199.

7) Kato, M. et al., Tohoku J. Exper. Med., 1953, 58, 143.

8) Matsuda, K. et al., Tohoku J. Exper. Med., 1955, 62, 221.

9) Aviado, D.M. et al., Am. J. Physiol., 1951, 165, 261.

10) McDowall, R.J.S., J. Physiol., 1924, 59, 41; ibid., 1934, 81, 5P; ibid., 1934, 83, 37P. 